\title{
Design of intelligent temperature control system for parking vehicle based on solar energy
}

\author{
Liu Xiaomin ${ }^{1}$, a , Tang $\mathrm{Jie}^{1}$, and Wang Zhaoming ${ }^{1}$ \\ ${ }^{1}$ College of Applied Technology, Jilin University, Changchun, China
}

\begin{abstract}
Because of the high summer temperature, after the vehicle parking, the interior temperature increased sharply. It can make the vehicle interior parts easy to age, and release harmful gases. While the driver can't adapt to high temperature when enter to the vehicle again. The interior high temperature is a threat to human's health. In order to reduce the temperature inside the vehicle, this paper designed a solar-based intelligent temperature control system. After the vehicle's engine stops working, it can monitor the temperature inside the vehicle in real time, and can reduce the temperature to the appropriate temperature when the temperature is too high. The system has the advantages of simple structure, convenient use, no need to modify the vehicle and so on. After the real vehicle test, the effect of reducing the heat accumulation in the vehicle can be achieved.
\end{abstract}

\section{Introduction}

\subsection{The hazards of high temperatures in vehicles}

In recent years, the amount of vehicle ownership has increased significantly in China, and vehicles have become an important means of transportation for people to travel. When driving in hot summer, the temperature inside the vehicle rises, and people use air conditioning to cool down. However, when the automobile stops working, the air conditioning has no power source. so it stops working. Under the strong radiation of the sun, the temperature inside the vehicle rises rapidly. McLaren and others had make an actual measurement on the temperature inside the vehicle, the result is that when the outside temperature is $22{ }^{\circ} \mathrm{C}$, the parked vehicle compartment temperature can reach $47{ }^{\circ} \mathrm{C}$. When the driver re-enters the vehicle to drive, the high temperature of the interior will make the driver very uncomfortable and it's not conducive to good health.

In addition, there are many hazards because of the high temperature in the vehicle after parking. In recent years, there have been many related news reports, such as children have been forgotten in the vehicle, the high temperature inside the vehicle caused brain damage and even death of children, as well as the vehicle placed perfume bottles, lighters and other items in the high temperature burst, the vehicle placed bottled beverages in the high temperature will produce toxic substances harmful to health, leather ornaments at high temperature will accelerate aging and many other problems.

\subsection{Research status and significance}

At present, there are two commonly adopted treatment methods. The first one is to open all the doors of the vehicle and use the air convection to reduce the temperature inside the vehicle. The temperature drops to the appropriate temperature, which need about at least 15 minutes or more.

The other method is to start the engine, open the vehicle air conditioning's most upscale forced cooling. This method is also need to wait for some time to make the vehicle temperature drop down. It has some disadvantages, such as the vehicle idle operation for a long time, will greatly increase the oil consumption, with the experimental data showed that oil consumption can be increased by $14.2 \%$. At the same time, when the vehicle is operating at idle, the fuel is not completely burned. So there's a lot of carbon around the valve. The vehicle will exhaust a large number of harmful discharges, which will pollute the environment. This is not conducive to environmental protection.

In recent years, much of the researches on the application of solar energy in vehicle cooling mainly are focus on how to connect solar cells to the air conditioning system in the vehicle. After the vehicle is parked, solar cells drive the air conditioning system to work to achieve the purpose of cooling, which requires the vehicle to be redesigned or modified.

In view of the above problems, it has important sense to design an intelligent temperature monitoring and cooling device based on solar energy, which can automatically reduce the temperature inside the vehicle without changing the automobile's original structures.

This device can make the driver feel comfortable 
when entering the vehicle, at the same time, it can extend the service life of the vehicle interior, and effectively reduce a series hazards caused by the high temperature of the vehicle.

\section{Overall design scheme of the system}

\subsection{Collection and analysis of environment temperature and interior temperature data}

In the summer, the vehicles are exposed to the sun after they stopped. Most of the vehicle body is metal, so the body will absorb heat and conduct heat. Under the sunshine, the body of the vehicle will gather a lot of heat, which will be conducted to the interior. The sun also shines through the windows into the inside of the vehicles. As the parking time increases, the temperature inside the vehicles will increase rapidly.

Figure 1 shows the measured environmental temperature in Changchun someday and the numerical curve of the temperature inside the vehicle. The test condition is that the doors and windows are closed and the front windshield is not shielded. The maximum environmental temperature is $33{ }^{\circ} \mathrm{C}$, the maximum temperature inside the compartment can reach $57^{\circ} \mathrm{C}$, and the maximum temperature difference between inside and outside the compartment can reach $26.9^{\circ} \mathrm{C}$. As can be seen from Figure 1, with the increase of the parking time, the temperature inside the compartment will continue to rise even if the range of environmental temperature change is not large.

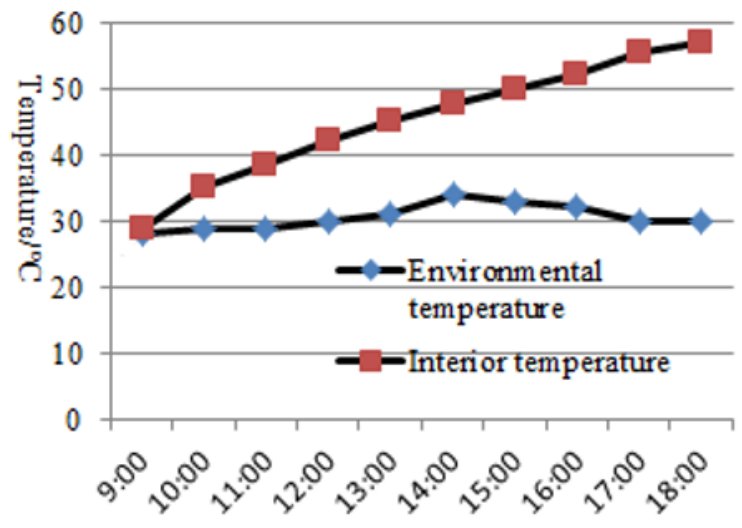

Fig1. The temperature curve of environment and interior

Due to the large area of the front windshield of the vehicle, more sunlight is shone into the vehicle through the front windshield. Therefore, the difference of the internal temperature change of the vehicle is compared and analyzed when the front windshield of the vehicle is shielded or not. The test conditions are as follows: After the vehicle is parking, the doors and the windows of the vehicle are shut. The front windshield of a car is shaded by a sun visor. Under these conditions, the temperature data in the vehicle is measured. The environment temperature and the temperature data inside the vehicle are shown in figure 2.The red bar chart represents the environment temperature, and the purple bar chart represents the vehicle temperature. The figure 2 shows that when the environmental temperature is at $30{ }^{\circ} \mathrm{C}$ or so, the vehicle temperature increased with the extension of the parking time. The highest temperature in the vehicle is more than $50{ }^{\circ} \mathrm{C}$.

As can be seen from the comparison between Figure 1 and Figure 2, when the environmental temperature is roughly the same, if the front windshield is shielded, the temperature inside the vehicle rises slowly and the maximum temperature decreases slightly. Therefore, shielding the front windshield is conducive to reducing the temperature inside the vehicle. Wang Weijian et al. studied on the influence of solar irradiation on the interior temperature, and measured the temperature in Guangzhou. When the environmental temperature is above $35^{\circ} \mathrm{C}$, the temperature near the dashboard of the interior vehicle can reach above $90{ }^{\circ} \mathrm{C}$. At the same time, when the front windshields have different colors, the dark color of the windshield can make the temperature rise slowly, which is consistent with the measured data in Changchun.

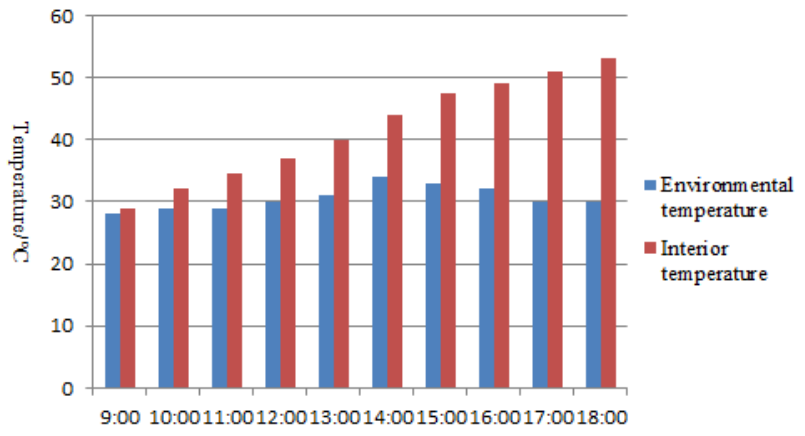

Fig2. The temperature of environment and interior measured after shielding the front windshield

Through the comparison of measured temperature data, shielding the front windshield has an effect on reducing the temperature inside the vehicle, so the cooling device is placed on the front windshield.

\subsection{Functions to be realized by the system}

In order to reduce the temperature in the vehicle after the vehicle is parked, the cooling device designed in this paper needs to achieve the following functions: first, to block the radiation from the front windshield of the sun, second, to use the fan to form the air flow inside and outside the vehicle, third, to use semiconductor refrigeration chips to produce cooling effect, fourth, to monitor the temperature in the vehicle compartment in real time, the engine stops working after the vehicle stops, at this time the power supply is solar panels. Solar panels have the advantages of low cost and environmental protection, as well as a certain degree of flexibility, convenient to place on the curved object.

\subsection{System hardware design}

\subsubsection{System structure}

The main hardware components of the system are solar 
panels, temperature control switch, temperature sensor, fan, semiconductor refrigeration, sunshade baffle, etc., which can be divided into two modules: temperature monitoring module and temperature regulation module. The overall control structure of the control system is shown in Figure 3.

A number of solar panels are placed in a sunshade, which is shown in figure 4 . The solar panels are pasted in the visor corrugated. In driving process, the sunshade is rolled up by a retracting device. Stick it to the top of the front windshield so that it doesn't block the view.

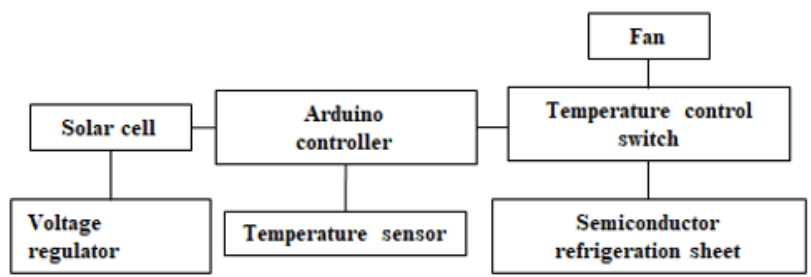

Fig3. Structure diagram of control system

When the vehicle is parking, the sunshade can be placed on the front windshield through the retractable device.

It can not only block the direct injection of sunlight, but also the electricity generated by the solar cell, through the temperature control switch to control the driving fan and semiconductor cooling chip work, form the inside and outside air circulation, to achieve the purpose of cooling.

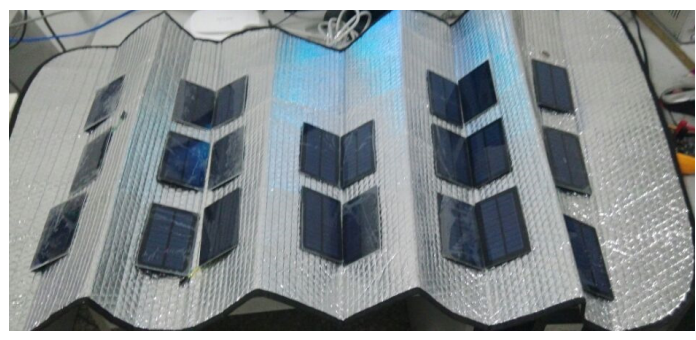

Fig4. Solar panel layout

\subsubsection{Working principle of the system}

The working principle of the solar cooling system designed in this paper is as follows: under the condition of sufficient sunlight, the solar panel receives sunlight to generate electricity and drives two small fans placed inside and outside the vehicle in reverse to realize the exchange of air, helping the flow of air inside and outside the vehicle to bring down the temperature inside the vehicle. The cooling system mainly consists of the following parts: signal acquisition system, control system, ventilation system and display system. Here's how they work:

The temperature sensor is powered by the solar cell, placed on the vehicle's instrument table, monitors the temperature in the compartment in real time, and transmits the temperature signal to the Arduino controller.

The control principle of the control system is shown in Figure 5: When the temperature in the compartment is less than $t_{1}\left(t_{1}\right.$ is defined as the starting temperature of the fan in low gear), the temperature control switch is kept long open and the temperature control system does not work; When the temperature in the compartment is as follows: $\mathrm{t}_{1}<\mathrm{t}<\mathrm{t}_{2}$ ( $\mathrm{t}_{2}$ is defined as the starting temperature of the fan in the high speed gear), the temperature control switch one (low speed gear) closed, and the temperature control system controls the rotation of the fan at low speed; When the temperature in the compartment is $t>t 2$, the temperature control switch two(high speed) is engaged. The temperature control system controls the high-speed rotation of the fan, and the semiconductor refrigeration unit starts to work at the same time.

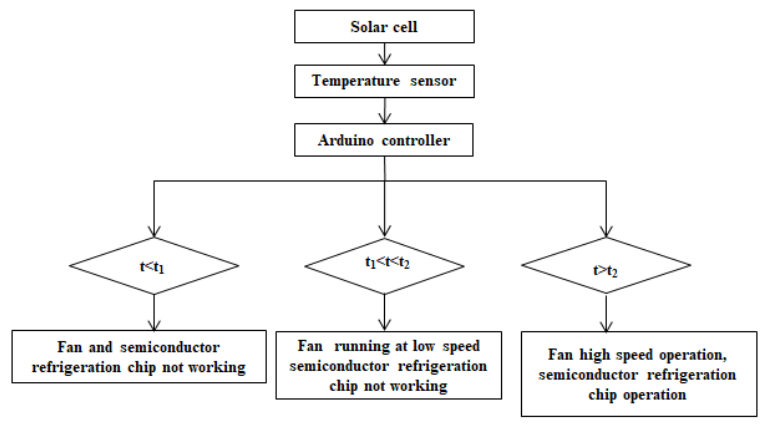

Fig5. Control schematic diagram of the control system

The system starting temperature $t_{1}$ and the fan switching temperature $t_{2}$ can be defined according to actual needs.

The ventilation system is mainly composed of a fan and a semiconductor refrigeration unit. The operating state of the semiconductor refrigeration unit is on and off. The running speed of the fan is controlled by the temperature control switch. According to the different temperature, the control system adjusts its working state.

The display system can display the real-time temperature in the compartment and the working status of the system.

\subsection{Software design of control system}

In order to realize a good temperature detection and adjustment function in the vehicle, an Arduino-based control system was designed and a software program was written. The workflow of the software system is as follows:

After the vehicle is in the parking state, we put adsorption cooling device on the front windshield. The solar cells will absorb sunlight, output voltage, and power supply to the system. When the driver press the startup command, the temperature sensor will collect signals of temperature in the vehicle, and then transfer it to the controller. The controller receives the signal, analysis the signal processing, and send instructions to the fan and the semiconductor refrigeration unit. The workflow of the software system is shown in Figure 6. 


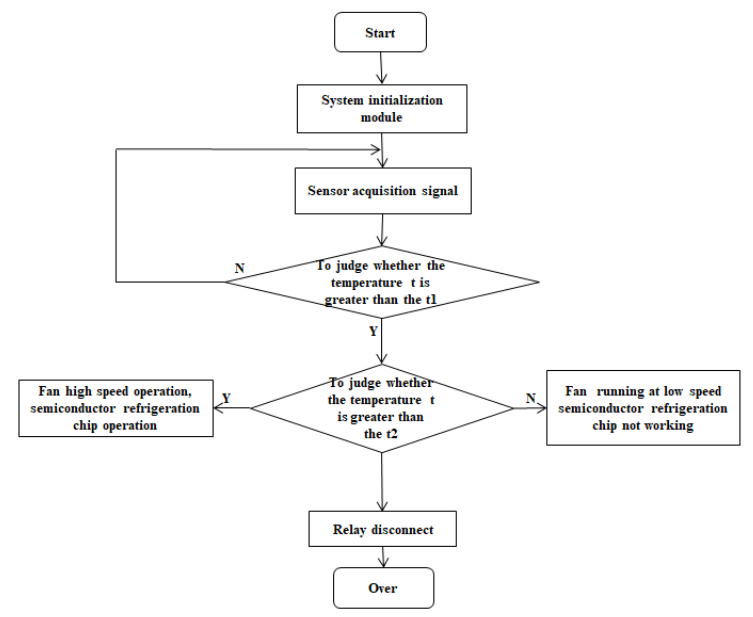

Fig6. The workflow of software system

\section{The analysis of system commissioning and real vehicle test}

The function of the cooling system was tested in real vehicle. The working state of the solar panel, the working shape of the temperature monitoring system and the working state of the temperature regulation system were tested respectively. The problems of unstable output voltage, temperature detection sensitivity and software program were solved. The real vehicle test vehicle is a hatchback vehicle, and the test picture is shown in Figure 7. The experimental scheme and results are as follows.

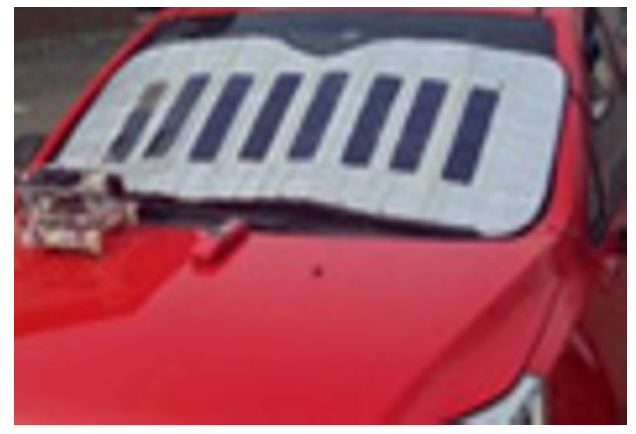

Fig7. Actual vehicle test diagram of the system

The test environmental conditions were as follows: the environmental temperature was $28{ }^{\circ} \mathrm{C}$, the starting temperature $\mathrm{t} 1$ of the refrigeration system was set as $40^{\circ} \mathrm{C}$, and the vehicle exposure value reached $35^{\circ} \mathrm{C}$ in the vehicle.

At this point, the working state of the test system is as follows: the output voltage of the solar cell is 12 volts; The temperature displayed on the temperature display is consistent with the thermometer placed in the vehicle, indicating that the temperature sensor is normal. The temperature in the vehicle did not reach the starting temperature of the refrigeration system of $40^{\circ} \mathrm{C}$, the fan and semiconductor refrigeration sheet did not work, and the system was in standby state, indicating that the hardware and software of the control system were working normally.

When the environmental temperature is $33^{\circ} \mathrm{C}$, test the system again. The system startup temperature $\mathrm{T} 1$ is set to $40^{\circ} \mathrm{C}$, the fan high-speed rotation startup temperature $\mathrm{T} 2$ is set to $50^{\circ} \mathrm{C}$, and when the vehicle is exposed to sunlight until the vehicle interior temperature is $46^{\circ} \mathrm{C}$, the fan operates at low speed, the semiconductor refrigeration unit does not work, and the interior temperature can be stabilized within $40^{\circ} \mathrm{C}$.

When the environmental temperature is $38^{\circ} \mathrm{C}$, test the system again. The starting temperature $\mathrm{t} 1$ of the system is set as $40^{\circ} \mathrm{C}$, and the starting temperature $\mathrm{T} 2$ of the highspeed rotation of the fan is set as $50^{\circ} \mathrm{C}$. When the vehicle is exposed to the sun until the temperature in the vehicle reaches $60^{\circ} \mathrm{C}$, the high-speed operation of the fan and the semiconductor refrigeration unit work, and the temperature in the vehicle can be stable within $40^{\circ} \mathrm{C}$.

Through the above tests on the cooling device in different ambient temperatures, it can be seen that the cooling system achieves the expected function and has a good cooling effect.

\section{Conclusions}

The real vehicle test results show that: the cooling system designed in this paper takes solar energy as the energy, using cooling curtain block part of the sun radiation. The solar cells can collect solar energy, output voltage, supply power to the system, and drive the inside and outside air circulation refrigeration through fans and semiconductor refrigeration chips, so as to realize the cooling effect of the vehicle and achieve the expected function.

In particular, the device is simple and reliable in design, easy to operate, safe and environmentally friendly. The suction cup can be attached to the front windshield of the vehicle without any modification of the vehicle, which has a wide application prospect.

\section{References}

1. C. McLaren, J. Null, J. Quinn. Heat stress from enclosed vehicles: moderate environmental temperatures cause significant temperature rise in enclosed vehicles [J].Pediatrics, 116:109-113(2005).

2. M. Korukcu, M. Kilic. Transient effects of different heating modes on temperature and humidity inside the automobile cabin during heating period[J]. Journal of Thermal Science and Technology, 32: 101-107(2012).

3. W. Weijian. Study on the influence of solar Radiation on interior temperature. Guangqi Honda Automobile Co. LTD.

4. W. Yinghua, X. Dan. Design and manufacture of automatic cooling system for solar energy vehicle in high temperature weather. Technology and Application: 135.

5. W. Xiaojie. The Study on the Cooling Performance of the Vehicle Cockpit Using the Parking Vehicle Air Conditioner Under Different Solar Radiation Conditions. Technological Innovation:87-91 (2019)

6. W. Gaopen, L. Miaoshan. The Applied Study on The 
Solar mechanical System in Automobile. Agricultural Equipment \& Technology.,37:25-28(2011)

7. D. Fengqi, L. Yuguo, H. Dingguo. Research on temperature limiting Device for heat dissipation inside parked vehicles. Journal of nantong university (natural science edition).12(4):52-56(2013)

8. Dadour I R, Almanjahie Iet al. Temperature variations in a parked Vehicle [J]. Forensic Science International, 207 : 205-211(2011).

9. A. Jamekhorshid, S M. Sadrameli. Application of phase change materials (PCMs) in maintaining comfort temperature inside an automobile $[\mathrm{J}]$. World Academy of Science: Engineering and Technology, 61 (1): 459-461(2012).

10. L. Chunmei. How to cool down quickly after exposure to the sun $[\mathrm{J}]$. Automotive applications, 201

11. M. Xuefeng, L. Xinglong. Portable refrigerator design based on solar Semiconductor refrigeration. Integrated Circuit application., 37(10):40-41(2020). 